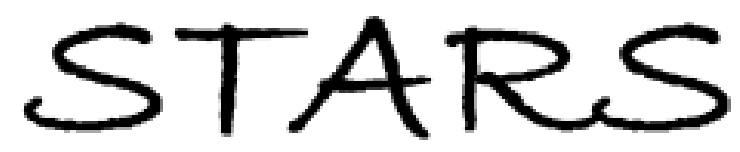

University of Central Florida

STARS

\title{
The Effects of Mindfulness Meditation on Stress and Burnout in Nurses
}

Alyssa Green

University of Central Florida

Find similar works at: https://stars.library.ucf.edu/honorstheses University of Central Florida Libraries http://library.ucf.edu

This Open Access is brought to you for free and open access by the UCF Theses and Dissertations at STARS. It has been accepted for inclusion in Honors Undergraduate Theses by an authorized administrator of STARS. For more information, please contact STARS@ucf.edu.

\section{Recommended Citation}

Green, Alyssa, "The Effects of Mindfulness Meditation on Stress and Burnout in Nurses" (2020). Honors Undergraduate Theses. 792.

https://stars.library.ucf.edu/honorstheses/792

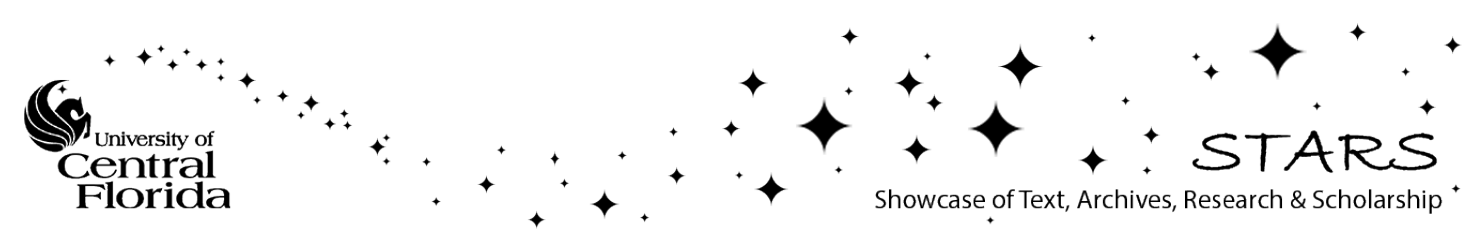




\title{
THE EFFECTS OF MINDFULNESS MEDITATION ON STRESS AND BURNOUT IN NURSES
}

by

\author{
ALYSSA ASHLEY GREEN \\ A thesis submitted in partial fulfillment of the requirements \\ for the Honors in the Major Program in Nursing \\ in the College of Nursing \\ and in the Burnett Honors College \\ at the University of Central Florida \\ Orlando, Florida \\ Summer Term \\ 2020
}

Thesis Chair: Elizabeth Kinchen, PhD 


\begin{abstract}
Occupational burnout related to stress in the workplace is frequently experienced by nurses who are regularly confronted with trauma, suffering, and high workloads. Burnout can negatively impact patient care and have detrimental effects on nurse's physical and mental health.

Mindfulness Based Stress Reduction programs have been researched as a potential intervention for reducing stress and burnout through cultivating present awareness, emotional regulation, and positive thinking. A literature review was performed to explore the current knowledge on the effectiveness of mindfulness meditation related to stress and burnout in nurses and to provide recommendations for future research on this topic.

Findings reveal strong evidence that mindfulness meditation is effective in decreasing stress and burnout in nurses. Mindfulness based interventions have been shown to significantly decrease stress, improve all aspects of burnout, and increase self-compassion and compassion satisfaction. Mindfulness meditation has the potential to prevent stress and burnout in nurses by decreasing self-judgement and over-identification with experience, and by increasing resiliency, compassion, and emotional regulation.
\end{abstract}




\section{DEDICATION}

For Cole: my guiding light, the keeper of my sanity, my teammate, and my best friend. Thank you for your never-ending support, and for introducing me to the practice of mindfulness.

For my mom and dad, who have always encouraged me to be the best that I can be and never failed to show me how proud they were at every step of the way.

For Christie, Richard, and Cassidy Hagerman, who have unknowingly taught me the importance of expressing daily gratitude. 


\section{ACKNOWLEDGEMENTS}

Thank you to my thesis chair, Dr. Elizabeth Kinchen, who guided me through this process while providing me with invaluable advice and encouragement, as well as my committee members Dr.

Victoria Loerzel and Dr. Joyce Burr. Thank you all for believing in me before I believed in myself, and for allowing me the opportunity to explore this research. I truly appreciate all of you.

Thank you to the College of Nursing and the Burnett Honors College for this opportunity. 


\section{TABLE OF CONTENTS}

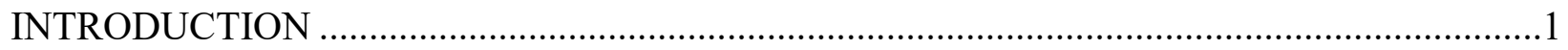

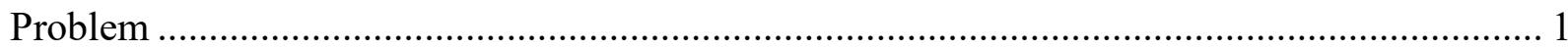

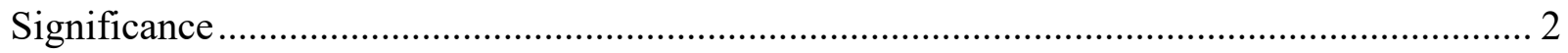

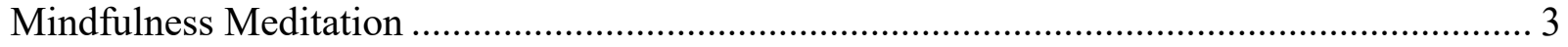

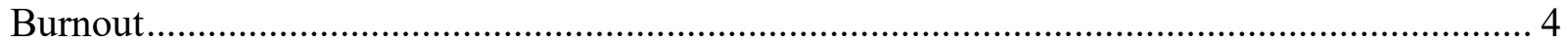

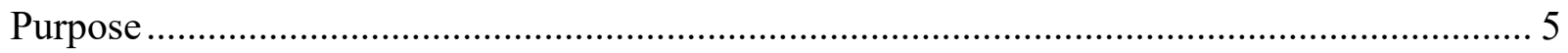

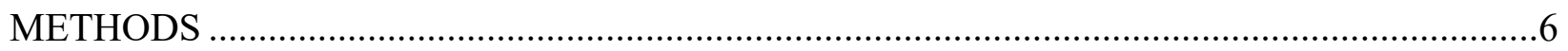

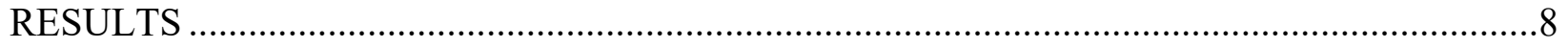

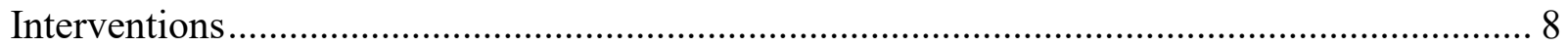

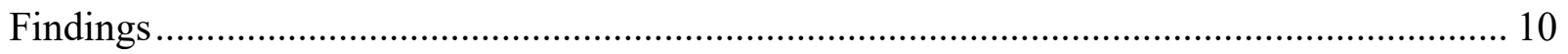

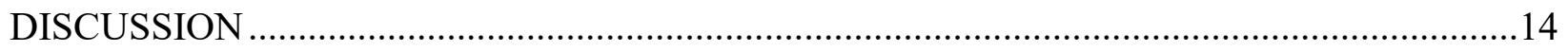

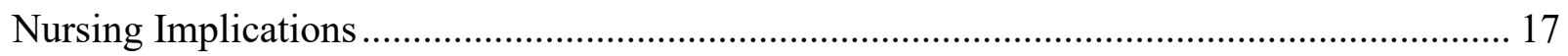

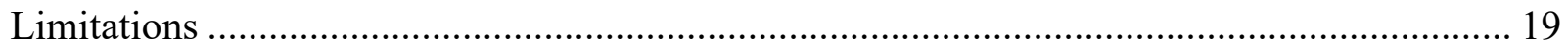

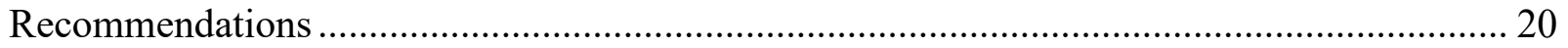

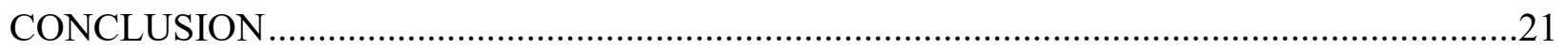

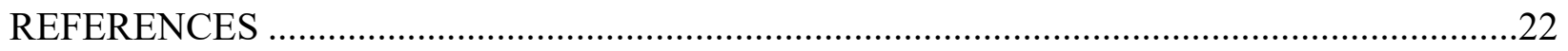




\section{LIST OF FIGURES}

Figure 1: Study Selection Process Flow Diagram .............................................................. 7

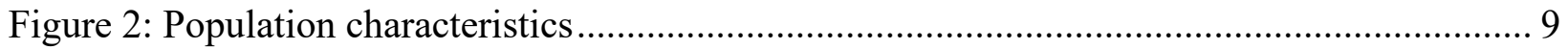




\section{INTRODUCTION}

\section{Problem}

Burnout is the result of high levels of stress caused by an inability to cope with or meet the demands of an environment. Nurses suffering from burnout are at risk for experiencing physical and psychological symptoms, such as fatigue, anxiety, depression, and overall job dissatisfaction (Flynn \& Ironside, 2017), and are more likely to abuse substances including caffeine, alcohol, and illicit drugs (Jarrad, Hammad, Shawashi, \& Mahmoud, 2018). Burnout has been shown to negatively impact an individual's relationships and home life, and it is associated with higher rates of physical illnesses such as hypertension, heart disease, sleep disorders, and influenza (Ferguson, Low, \& Shiau, 2020).

Research has shown that dissatisfied, disinterested, and overworked nurses can have negative impacts on patient outcomes and their level of satisfaction (Vahey et al., 2004) as a result of a decreased quality of care (Flynn \& Ironside, 2017). Inadequate staffing has been linked to inpatient mortality and adverse events for patients, including falls and medication errors. (Spence Laschinger \& Leiter, 2006). Understaffing also contributes to 'time-pressure', or stress that occurs when one has less time than needed, which was found to reduce the tendency of nurses to provide appropriate interventions (Thompson et al., 2008), and a study in Oman found that high levels of burnout were indicators for a reduction in perceived patient safety among critical care nurses (Ma'mari, Sharour \& Omari, 2020).

Burnout among nurses can have long-term negative effects on the hospitals that employ them. Research has shown that higher levels of burnout could potentiate rates of sick leave and absenteeism (Parker \& Kulik, 1995), as well as tardiness and turnover (Vahey et al., 2004). 
These factors can contribute to the overworking of staff members, further increasing the likelihood of nurses experiencing burnout. High levels of turnover and absenteeism can be costly for healthcare organizations that find themselves hiring and training new employees, in addition to overtime pay to existing employees when understaffing is an issue. Burnout has been noted as one of the main contributing factors to attrition, a gradual decrease in effectiveness among healthcare professionals (Flynn \& Ironside, 2017).

\section{Significance}

Prevention of burnout in nurses is aimed at promoting personal wellbeing and the development of coping mechanisms for occupational stress (Kravits, McAllister-Black, Grant \& Kirk, 2010). The Mindfulness-Based Stress Reduction (MBSR) program, developed by Dr. Jon Kabat-Zinn in the 1970s, consists of an 8-week course where participants engage in guided meditations focused on cultivating nonjudgmental awareness. Studies aimed at determining the effectiveness of the MBSR program have found that participants have experienced reduced burnout, stress, depression, anxiety, and compassion fatigue, as well as increased life satisfaction, self- compassion, and empathy (Ghawadra, Abdullah, Choo \& Phang, 2019).

A study performed in the Midwest found that after eight weeks, the healthcare provider participants reported significantly improved levels of stress, non-reactivity to inner experience, and quality of life (Benzo, Anderson, Bronars, \& Clark, 2018). Nurses working at an adolescent mental health facility reported reduced levels of stress and absenteeism after completing the standard 8-week MBSR program (Hallman, O’Conner, Hasenau \& Brady, 2014). Nursing students have also seemed to benefit from such programs. One study found that in addition to reduced stress, the student participants reported increased patience, appreciation, and acceptance 
(van der Riet, Levett-Jones, \& Aquino-Russel, 2018). A study in Brazil found that nurses who completed the MBSR had significant reductions in burnout, specifically exhaustion and depersonalization, for at least three months after completing the program. The same participants also reported feeling less reactive and felt greater empathy towards patients and in their personal relationships (dos Santos et al., 2016). Research has shown that MBSR programs have significantly reduced psychological distress in a variety of populations, including patients with breast cancer and vascular disease, veterans, university students (Ghawadra et al., 2019) in addition to nurses. (Aikens et al., 2014).

Improvements in levels of stress may have a profound impact on reducing burnout in nurses and should be explored as a potential intervention for improving satisfaction, personal achievement, depersonalization, emotional exhaustion, and other negative consequences of occupational burnout.

\section{Mindfulness Meditation}

Meditation is defined as an engagement in contemplation or reflection and is traditionally known as an integral part of Buddhism, which aims to seek wisdom from expanding awareness and developing compassion. There are many subcategories of meditation, each with distinct traditions, guidance, and methods of practice. Vipassanā, the Pali word for "special seeing" or "insight," began in Burma in the 1950s, and is the central concept of mindfulness meditation. Practicing mindfulness involves active participation in the present moment and examination of the nature of reality. Joan Borysenko, the president of Mind/Body Health Sciences Inc., describes mindfulness as "a way of life that reveals the gentle and loving wholeness that lies at the heart of our being, even in times of great pain and suffering" (Kabat-Zinn, 2013, p.13). 
Szekeres (2015) describes mindfulness as a shift in perspective and a detachment to sensations and thought, allowing one to become non-reactive to aversions (the desire to avoid an unpleasant sensation) and cravings (the desire to prolong pleasant sensations). Aversions and cravings promote stress and unhappiness (Szekeres \& Wertheim, 2015) by taking one out of the present moment and judging the current experience as "good" or "bad." Similarly, postulating about the future and ruminating about the past may promote stress and contribute to unhealthy coping mechanisms (Baer, Smith, \& Allen, 2004). The practice of mindfulness aims to maintain a nonjudgmental view of experience, whether it be internal thought and emotion, or external stimuli such as sights and sounds (Baer, 2006). Through repeated acceptance of unpleasant sensation and nonjudgmental observation of the present, the individual may be better equipped to find the balance between environmental demands and the ability to cope with stress.

\section{Burnout}

Chronic exposure to psychological stress resulting from an imbalance between occupational demand and an individual's ability to cope is known as 'burnout' (Saeidi, Izanloo, \& Izanlou, 2020). A growing number of healthcare professionals are finding themselves unable to cope with high levels of stress, with studies reporting that over $40 \%$ of hospital nurses and up to $70 \%$ of Intensive Care Unit nurses experience work-related burnout (Vahey, Aiken, Sloane, Clarke \& Vargas, 2004; van Mol, Kopanje, Benoit, Bakker, \& Nijkamp, 2015).

This phenomenon is characterized by emotional exhaustion, depersonalization, and decreased personal accomplishment (Maslach \& Jackson, 1981). Burnout is often a gradual process wherein the individual's physical and emotional capabilities are diminished after prolonged exposure to stress in the workplace. The high demands of the healthcare system are 
often out of an individual's control. High workloads, lack of support, constrained resources, the emotional nature of the job, and inadequate staffing are major contributing factors (Flynn \& Ironside, 2017; Spence Laschinger \& Leiter, 2006).

\section{Purpose}

The purpose of this literature review is to explore the current knowledge on the effectiveness of mindfulness meditation related to stress and burnout in nurses. In addition, this thesis will examine gaps identified in the current literature and provide recommendations for further research on this topic. 


\section{METHODS}

This review of literature utilized CINAHL, PsycInfo, MEDLINE, and ERIC to identify scholarly sources published in academic journals. Search terms included mindfulness,

meditation, MBSR, occupational stress, stress, burnout, and nurs*. Inclusion criteria consisted of research directly related to the impact of mindfulness on nurses who are experiencing stress and/or burnout, and peer reviewed articles published after 2000 in the English language. Literature examining the impact of mindfulness on healthcare professionals other than nurses was excluded. The sample $(n=9$ was obtained after careful review of potentially relevant material and discarding literature which was inaccessible, redundant, or did not fit the inclusion criteria. The selection process is described in more detail in Figure 1. 
Key search terms: mindfulness, meditation, MBSR, nurs*, stress, occupational stress, burnout Limiters: English, peer reviewed, published after 2000

Potentially relevant results after initial search of databases (CINAHL, APA PsycInfo, ERIC, MEDLINE)

$$
(n=368)
$$

Studies excluded for not meeting inclusion criteria $(n=287)$

Studies retrieved for detailed review

$$
(n=81)
$$

Studies excluded for not meeting inclusion criteria $(n=74)$

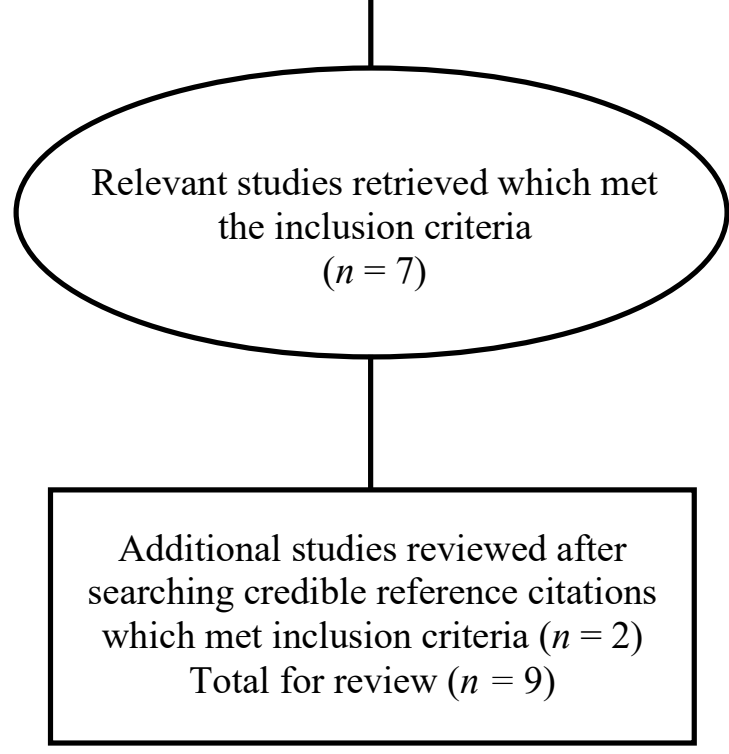

Figure 1: Study Selection Process Flow Diagram 


\section{RESULTS}

\section{Interventions}

This review of literature examines nine studies involving mindfulness meditation interventions in nurses across various disciplines, including oncology, pediatrics, intensive care, and general hospital settings. Each study was performed in a different country across varying cultures, and one study took place in both Israel and the United States. Most studies had a sample size between 10 to 40 nurses $(n=6)$, and the largest sample size was $n=82$. A detailed description of populations can be found in Figure 2. The participating nurses in all studies were mostly female and had varying amounts of experience, however all studies found that sociodemographic data did not have a significant impact on results.

The Mindfulness-Based Stress Reduction (MBSR) developed by Jon Kabat-Zinn (1990) serves as the blueprint for many modern mindfulness programs. Traditional MBSR requires a lengthy time commitment, as it comprises 2.5 -hour weekly group sessions and 45-minute daily meditations over 8 weeks, in addition to 1 full-day retreat lasting 6 hours. The program focuses on increasing mindfulness and attention through various techniques, including formal sitting practice and mindful movement. Three of the nine studies reviewed used the traditional MBSR approach, while the remaining studies used either a modified MBSR program or a nontraditional program.

The majority of interventions took place over 8 weeks $(n=6)$. The lengthiest intervention was an 8-month program $(n=1)$, while the shortest was 4 weeks $(n=2)$. Most of the interventions were group-based $(n=7)$, meaning participants met weekly to practice together with the guidance of a trained instructor. In addition, participants were given CDs with pre-recorded meditations 
ranging from 10-45 minutes and encouraged to practice at home. The remaining studies $(n=2)$ were completed individually and required participants to complete pre-recorded meditations 5 to 7 days a week. Five studies included either a full-day retreat, half-day retreat, or an extended final group session.

\begin{tabular}{|c|c|c|c|}
\hline Author(s), Year & Sample size & Population & Country \\
\hline $\begin{array}{l}\text { Daigle, Talbot, \& } \\
\text { French, } 2018\end{array}$ & $n=37$ & General hospital setting & Canada \\
\hline Delaney, 2018 & $n=13$ & $\begin{array}{l}\text { Oncology, cardiology, urology, } \\
\text { maternity, midwifery, } \\
\text { intensive care }\end{array}$ & U.K. \\
\hline $\begin{array}{l}\text { Gracia Gozalo et al., } \\
2017\end{array}$ & $n=13$ & ICU & Spain \\
\hline Hevezi, 2016 & $n=17$ & Oncology & U.S. \\
\hline $\begin{array}{l}\text { Lin, He, Yan, Gu, \& } \\
\text { Xie, } 2018\end{array}$ & $n=55$ & General hospital setting & China \\
\hline Moody et al., 2013 & $n=24$ & Pediatric oncology & Israel \& U.S. \\
\hline $\begin{array}{l}\text { Sallon, Katz-Eisner, } \\
\text { Yaffe, \& Bdolah- } \\
\text { Abram, } 2017\end{array}$ & $n=82$ & General hospital setting & Jerusalem \\
\hline $\begin{array}{l}\text { Slatyer, Craigie, } \\
\text { Heritage, Davis, \& } \\
\text { Rees, } 2018\end{array}$ & $n=65$ & General hospital setting & Australia \\
\hline Wang et al., 2016 & $n=31$ & $\begin{array}{l}\text { Newly graduated/newly } \\
\text { employed }\end{array}$ & Taiwan \\
\hline
\end{tabular}

Figure 2: Population characteristics

The studies in this literature review examined mindfulness meditation's effect on burnout $(n=6)$ and stress $(n=4)$, in addition to other experiences that result from chronic workplace stress and may be synonymous or closely related to burnout. These factors include tension, anxiety, depression, and secondary traumatic stress, which may result from acute or chronic exposure to the suffering of others and is frequently seen in nurses who are experiencing burnout (Delaney, 
2018). One study examined the effect of mindfulness on nursing errors. In addition, some studies looked at mindfulness meditation's effect on factors that may be protective against burnout, including self-compassion, resilience, and compassion satisfaction, which is the positive feeling of providing competent and compassionate care (Hevezi, 2016). During the post-intervention phase, some researchers asked participants to identify the perceived benefit of the program on their personal life, wellbeing, and job satisfaction.

Most of the studies reviewed $(n=7)$ used consistent methods of measurement, utilizing the Maslach Burnout Inventory (Maslach \& Jackson, 1981), Self-Compassion Scale (Neff, 2003), Perceived Stress Scale (Cohen, Kamarck, \& Mermelstein, 1983), or the Professional Quality of Life Scale (Stamm, 2012). All measures used were evaluated for validity, reliability, and international use. Together, these measures provide quantitative data on participant's levels of burnout, stress, compassion satisfaction, and secondary traumatic stress.

\section{Findings}

A study conducted by Daigle, Talbot \& French (2018) found that nurses participating in a modified MBSR program had a significant decrease in work-related distress after 2.5-hour sessions over 8 weeks, along with a retreat. The sessions were focused on increasing attention and mindfulness through formal sitting practice as well as body scans, yoga, and mindful walking. More than double the nurses who received the intervention experienced at least a $50 \%$ reduction in distress when compared to nurses in the control group receiving no intervention. Almost all participants (96.4\%) reported feeling as though it was worthwhile to continue the practice after the programs conclusion, and $75 \%$ of participants rated the perceived benefit on their work performance as at least an 8 out of 10 . Furthermore, 3 of the 8 participants who 
admitted to making errors at work before the intervention reported a decreased frequency in errors over 3 months post-intervention.

Newly graduated or newly employed nurses participating in a traditional MBSR program also experienced decreased levels of stress, which persisted through the following 6 months (Wang, 2017). Interestingly, levels of stress increased after 4 weeks, but decreased to a new low by the end of the intervention. Since this study examined young nurses between the ages of 23 to 24 with little experience, it is possible that the stress of their new career combined with their commitment to participating in a research program could be responsible for this increase. Despite this, levels of mindfulness steadily increased over the course of the program.

Pre-intervention burnout scores indicated that all participants in a study of pediatric oncology nurses had high degrees of depersonalization and diminished personal accomplishment, in addition to significantly higher levels of perceived stress than the U.S. national average (Moody et al., 2013). The intervention included an introduction session, 6 weekly sessions, and a final group session, for a total of 15 hours of formal group practice over 8 weeks. Sessions focused on awareness of body, thought, and emotions, identifying personal biases, developing listening and communication, exploring self-care, and noticing reactions to stress. Post-intervention, there were almost no changes in burnout or stress. However, all participants described experiencing perceived benefit from the program, including increased inner peace, decreased stress and anxiety, increased self-awareness, anger management, improved relationships, and improved focus. Moreover, nurses from this group also reported using mindfulness techniques such as "STOP” (Stop, Take a breath, Observe current state of mind and body, Proceed) at work when preparing for stressful situations. 
Researchers in China developed a modified 8-week MBSR program consisting of 2-hour weekly group sessions and 20-minute independent meditations at least 6 days a week (Lin, He, Yan, Gu, \& Xie, 2018). Nurses participating in this study experienced statistically significant improvements in stress at the end of the 8-week program which was sustained at the follow-up 3months post-intervention. Participants in this study saw no improvements in resilience at the conclusion of the program, however significant improvements in resilience were seen at the 3month follow-up. This delayed improvement may suggest that mindfulness practice can gradually affect other coping skills through increased emotional regulation and decreased overidentification during stressful events.

Gracia-Gozalo et al. (2017) examined a sample of 13 nurses working in an intensive care unit. The study used a virtual program which consisted of weekly meditations, each 5-8 minutes in length, delivered via WhatsApp in audio format along with additional information regarding the practice and daily reminders. Participating nurses experienced a significant positive effect in the emotional exhaustion aspect of burnout, which was moderate at baseline, as well as a significant increase in self-compassion. Participants were "very satisfied" with the intervention and expressed a desire to continue practicing.

Slatyer, Craigie, Heritage, Davis, \& Rees (2018) developed the Mindful Self-Care and Resiliency (MSCR) Intervention to reduce burnout and increase compassion satisfaction, selfcompassion, and resilience in nurses. The program featured educational workshops on compassion fatigue resiliency and mindfulness over 4 weeks, in addition to required practice at home. Results included significant improvements in compassion satisfaction, depressed mood, self-compassion, subjective quality of life, and burnout. Reduced levels of burnout remained 
unchanged by the 6-month follow-up. Another study with a 4-week intervention included daily independent 10-minute meditations and found that nurses experienced statistically significant improvements in compassion satisfaction and burnout (Hevezi, 2016). Participants reported increased relaxation and self-compassion, improved reactions to stress, and a high likelihood of continuing to practice meditation. Furthermore, participants from this study said they practiced breathing exercises from the intervention at the bedside and reported feeling less stressed while at work.

Several studies have used a multimodal approach to combatting stress and burnout. The Mindful Self-Compassion training program (Delaney, 2018) had participants practice mindfulness meditation along with loving-kindness meditation and compassion meditation over the course of 8 weeks. Sallon, Katz-Eisner, Yaffe, and Bdolah-Abram (2017) conducted a pilot study which allowed participants to partake in various mindfulness techniques including mindfulness meditation and mindful relaxation, in addition to drawing, journaling, dance, and acupressure. By offering a variety of options for cultivating mindfulness, these studies hoped to provide nurses with a foundation that enabled personal development of healthy coping mechanisms. The Mindful Self-Compassion program produced improvements in selfcompassion, mindfulness, compassion satisfaction, resilience, and burnout, while Sallon et al.'s (2017) pilot study showed improvements in emotional exhaustion and perceived stress. Common complaints within the Mindful Self-Compassion program included falling asleep and resistance to practice (Delaney, 2018), although these qualities were rated as least importance. Positive mental states and enhanced coping were rated as most important. Together, these studies identify alternative ways to enhance wellbeing though mindfulness and improve stress and burnout. 


\section{DISCUSSION}

The current research provides strong evidence that mindfulness meditation is effective in decreasing stress and burnout in nurses. Mindfulness-based interventions have been shown to significantly decreased stress (Sallon et al., 2017), improved all aspects of burnout (Delaney, 2018; Gracia Gozalo et al., 2018; Hevezi, 2016; Sallon et al., 2017; Slatyer et al., 2018), and increased self-compassion and compassion satisfaction (Hevezi, 2016; Slatyer et al., 2018). Participating nurses have reported a decreased frequency in errors (Daigle, 2018) and have seen positive effects on their physical and mental health (Sallon et al., 2017).

While some studies found that mindfulness produced nonsignificant results or no effect on certain variables, other studies found significant or positive results in the same variables. This may reflect the multifaceted nature of workplace stressors between units and the varying degree of exposure to trauma and suffering, and suggests that mindfulness interventions should be implemented with the demographics and specific needs of the population in mind. This may be accomplished by administering surveys, such as the Maslach Burnout Inventory or SelfCompassion Scale, to establish baseline characteristics, existing protective factors, and potential areas of improvement. For example, Delaney (2018) found that high levels of self-compassion, resilience, and mindfulness in a population could predict lower levels of burnout, while low levels of self-compassion, resilience, and mindfulness were positive predictors of increased frequency of burnout. A population exhibiting low levels of self-compassion and resilience might benefit more from a mindfulness program targeted specifically at these characteristics, such as the Mindful Self-Compassion and Resilience program demonstrated in Slatyer et al.'s

study (2018). Otherwise, self-directed or generalized mindfulness programs may be better suited 
for populations with high levels of protective factors as a prophylactic intervention or to maintain these traits.

Mindfulness based interventions are typically well-received and have demonstrated strong sustainability. Post-intervention surveys indicated that participants were interested in continuing to practice mindfulness after the programs concluded and found the skills they were learning had a positive impact on their work and personal lives (Gracia Gozalo et al. 2017; Daigle, 2018, Moody et al., 2013; Hevezi, 2016). Sallon et al., (2017) found that in an intervention lasting 8 months, attendance was high and rates of attrition were low. $94 \%$ of the participants in their study said the intervention improved their ability to cope with workplace stress, and 99\% reported using stress reduction techniques from the program outside of work. In addition, 91\% said they would consider joining another program in the future, and 94\% said they would recommend the program to a colleague. Another study found that $100 \%$ of participants said they were either "pretty likely" or "definitely likely" to continue mindfulness meditation (Hevezi, 2016).

Despite finding that high levels of burnout and stress remained unchanged after intervention in pediatric oncology nurses, participants still reported a significant perceived benefit from the program and the practice of mindfulness (Moody et al., 2013). Prior to the intervention, these nurses described their experience with burnout as feeling overwhelmed with high workloads, ineffective on patient outcomes, and physical discomfort. They also felt that their workplace stress affected their home life, caused rumination on negative thoughts, and made it difficult to sleep. After the program, nurses were able to use the techniques they learned to feel calm and relaxed, detach themselves from angry patients in order to resolve problems, and 
provide relief from physical pain. Some of the participants viewed the meditation class as an additional task to add to their already busy day, and felt the program increased their stress. However, journal entries revealed that this additional stress decreased as the program progressed. Some researchers believe the extended time commitment of traditional MBSR could be a deterrent for healthcare professionals (Lin, 2018). Nurses are often working 12-hour shifts and may find it difficult to commit to the practice of meditation long-term, especially if they are already experiencing stress and burnout. Preliminary inquiry of progressive care unit nurses' interest in mindfulness revealed preconceived beliefs that meditation is time consuming and difficult (Hevezi, 2016). However, research has shown that as little as 5 to 10 minutes of meditation a day over 4 to 8 weeks can decrease compassion fatigue and emotional exhaustion, and improve self-compassion and compassion satisfaction (Hevezi, 2016; Gracia Gozalo et al., 2017).

Mindfulness requires careful attention to the present moment, thereby decreasing ruminative thoughts and anxiety. Careful attention to the present moment can also promote a safer work environment and improve patient care by reducing distraction-induced mistakes. Mindfulness can increase the ability to cope with stress and difficult situations by allowing the individual to examine their experience without judgement or attachment. This nonjudgmental perspective promotes a positive, compassionate relationship with the self, which can extend others and improve relationships with loved ones, patients, and coworkers.

Mindfulness is accessible, cost-effective, and able to be practiced at one's discretion. Mindfulness is not limited to formal sitting meditation, and programs typically encourage participants to be mindful while performing everyday activities, such as eating, walking, or while 
at work, increasing the potential flexibility of their self-care routines. Formal lessons with a trained meditation guide are not a requirement for success, as demonstrated by Hevezi (2016) and Gracia Gozalo et al., (2017), and mindfulness does not necessitate the purchase of additional materials. Furthermore, study participants had an overall positive response to the programs and maintained high attendance rates, suggesting that nurses who begin practicing mindfulness find it enjoyable, relevant and beneficial. These benefits, along with the lack of negative consequences of mindfulness meditation, suggest that mindfulness meditation should be encouraged to prevent and decrease stress and burnout in nursing.

\section{Nursing Implications}

Once one has practiced mindfulness meditation and learned how to be mindful, it can be used anywhere, at any time. Nurses working in a pediatric ICU described feeling more confident, focused, self-aware, and able to handle difficult situations at work and at home (Moody et al., 2013). These nurses attributed these changes to the practice of techniques learned in the intervention program such as self-reflection, self-awareness, body scans, and breathing exercises. The "STOP" method taught in this program requires one to pause for a moment, take a breath, and become aware of the current mind and body experience before proceeding. This method can easily be used before entering a patient's room and may allow nurses to be more focused and aware on the situation.

Working on the frontlines of healthcare puts nurses at risk for frequent exposure to the suffering, stress, and negative emotions of others. Nurses may find that mindfulness allows them to disengage from stress by decreasing the sense of over-identification with experience. By reducing reactivity to stressful or difficult situations, mindful nurses may find themselves feeling 
more relaxed and less susceptible to negative emotions. Nurses reported using mindfulness as a preventative measure against anxiety, agitation, and exhaustion while at work (Delaney, 2018), which could increase their ability to be compassionate, calm caregivers. In one study, over half of the participants said they used mindfulness when working with patients and colleagues (53\% and $51 \%$, respectively) and $95 \%$ of participants said the mindfulness techniques they learned were relevant to the type of stress encountered at work (Sallon et al., 2017).

Meditation can be beneficial when practiced in group settings or individually, giving nurses the freedom to decide which setting they find most effective and allowing them to be flexible with their self-care routine. Independent practice has been shown to significantly increase self-compassion and have a positive effect on emotional exhaustion, personal achievement, and reactions to stress (Gracia Gozalo et al., 2017; Hevezi, 2016). Therefore, units may benefit from offering introductory information sessions to provide nurses with a foundation for individual practice. However, formal group programs may have the added benefit of producing the positive emotions associated with social support, which could strengthen relationships and improve teamwork among nurses on a unit. Some nurses felt that group discussions were beneficial and made them feel validated, and they enjoyed being able to use the techniques with colleagues participating in the same program (Moody et al., 2013).

When deciding on a mindfulness program, it is important to assess the population's strengths, weaknesses, and risk factors to determine the efficacy and primary focus of the intervention. A unit with a large number of nurses who score low in self-compassion may be at risk for widespread burnout and should consider implementing a formal group program, whereas a unit scoring high in self-compassion and mindfulness might consider investing in an 
independent option for individuals to enroll in during particularly stressful times.

Nurses reported experiencing an increased sense of wellbeing for an average of 33 hours after participating in a mindfulness meditation class and described feeling "energized", "happy", and "relaxed" (Sallon et al., 2017). This suggests that it may be beneficial for units or teams to dedicate time before their shift to formal meditation. Throughout their shift, nurses can practice breathing exercises, active listening, and mindful walking to increase their awareness of the present moment.

\section{Limitations}

Several limitations can be noted within the literature. Despite significant findings, all of the studies had small sample sizes, with most of the studies having a sample between 10-40 nurses. This makes the results difficult to generalize. The studies reviewed lacked consistency in their interventions. While some were modeled after the traditional MBSR program, each intervention had varying methods in regard to length of meditations, required amount of practice each week, and program length. It is yet to be determined which intervention method is most effective across populations.

Finally, the self-regulated nature of the interventions and the voluntary recruitment of participants allows for the possibility of healthy user bias and healthy adherence effect, which is the increased likelihood of practicing other healthy behaviors outside of the study (Shrank, Patrick, \& Brookhart, 2011). This could suggest that those who choose to actively participate in mindfulness-based stress reduction programs are more likely to practice healthy self-care routines. It is possible that the nurses who take interest in these studies could be predisposed to self-reflection and self-compassion, and thus have a greater ability to avoid burnout. 


\section{Recommendations}

Further research on the effects of mindfulness meditation on stress and burnout in nurses is warranted, using large and more diverse samples to enhance the degree to which findings can be generalized. Future longitudinal studies should be conducted to determine the long-term efficacy of mindfulness meditation on nurses. Finally, research should be conducted on the feasibility of implementing mindfulness programs into the workplace as a self-care intervention for nurses. 


\section{CONCLUSION}

Nurses experiencing burnout can negatively impact patient care through emotional exhaustion and depersonalization, thereby diminishing their ability to connect with patients and deliver high-quality care. Similarly, burnt-out nurses can affect other members of their team through absenteeism, which increases the workload and stress of the unit. Mindfulness meditation has the potential to prevent stress and burnout in nurses by decreasing self-judgement and overidentification with experience, can increase resiliency, compassion, and emotional regulation. Mindfulness meditation can be available to anyone at any time or place, making it a cost-effective, flexible, and psychologically beneficial practice for nurses who are suffering from occupational stress and burnout. 


\section{REFERENCES}

Al Ma'mari, Q., Sharour, L. A., \& Al Omari, O. (2020). Fatigue, burnout, work environment, workload and perceived patient safety culture among critical care nurses. British Journal of Nursing, 29(1), 28-34. https://doi.org/10.12968/bjon.2020.29.1.28

Alharbi, J., Jackson, D., \& Usher, K. (2020). Personal characteristics, coping strategies, and resilience impact on compassion fatigue in critical care nurses: A cross-sectional study. Nursing \& Health Science, 22(1), 20-27. doi: 10.1111/nhs.12650

Barnard, L. K. \& Curry, J. F. (2011). Self-compassion: conceptualizations, correlates, \& interventions. Review of General Psychology 15(4), 289-303. doi: 10.1037/a0025754289

Boyle, D. A. (2015) Compassion fatigue: The cost of caring. Nursing, 45(7), 48-51. doi: 10.1097/01.NURSE.0000461857.48809.a1

Cohen, S., Kamarck, T., \& Mermelstein, R. (1983). A global measure of perceived stress. Journal of Health and Social Behavior, 24, 385-396. doi:10.2307/2136404

Daigle S, Talbot F, French DJ. Mindfulness-based stress reduction training yields improvements in wellbeing and rates of perceived nursing errors among hospital nurses. Journal of Advanced Nursing, 74, 2427-2430. https://doi.org/10.1111/jan.13729

Delaney, M. C. (2018). Caring for the caregivers: Evaluation of the effect of an eight-week pilot mindful self-compassion (MSC) training program on nurses' compassion fatigue and resilience. PLoS ONE, 13(11), e0207261. https://doi.org/10.1371/journal.pone.0207261 
Ferguson, C., Low, G., \& Shiau, G. (2020). Dunlop, J. (2015). Burnout in Canadian radiology residency: A national assessment of prevalence and underlying contributory factors. Canadian Associations of Radiologies Journal, 71(1): 40-47. doi: $10.1177 / 0846537119885672$

Flynn, L., \& Ironside P. M. (2017). Burnout and its contributing factors among midlevel academic nurse leaders. The Journal of Nursing Education, 57(1): 28-34. doi: $10.3928 / 01484834-20180102-06$

Germer, C. K. \& Neff, K. D. (2013). Self-compassion in clinical practice. Journal of Clinical Psychology, 69(8), 856-876. https://doi.org/10.1002/jclp.22021

Gracia-Gozalo, R. M., Ferrer Tarrés, J. M., Ayora Ayora, A., Alonso Herrero, M., Amutio Kareaga, A., \& Ferrer Roca, R. (2017). Application of a mindfulness program among healthcare professionals in an intensive care unit: Effect on burnout, empathy, and selfcompassion. Medicina Intensiva, 43(4), 207-216. Doi: 10.1016/j.medin.2018.02.005

Hevezi, J. A. (2016). Evaluation of a meditation intervention to reduce the effects of stressors associated with compassion fatigue among nurses. Journal of Holistic Nursing, 34(4), 343350. https://doi.org/10.1177/0898010115615981

Jarrad, R., Hammad, S., Shawashi, T., \& Mahmoud, N. (2018). Compassion fatigue and substance use among nurses. Annals of General Psychiatry, 17(1), 13. https://doi.org/10.1186/s12991-018-0183-5

Kabat-Zinn J. (1990). Full catastrophe living: Using the wisdom of your body and mind to face stress, pain, and illness. Dell Publishing. 
Lin, L., He, G., Yan, J., Gu, C., \& Xie, J. (2018). The effects of a modified mindfulness-based reduction program for nurses. Workplace Health \& Safety, 67(3), 111-122. https://doi.org/10.1177/2165079918801633

Luken, M. \& Sammons, A. (2016). Systematic review of mindfulness practice for reducing job burnout. American Journal of Occupational Therapy, 70(2), 1-10. doi: 10.5014/ajot.2016.016956

Maslach, C., \& Jackson, S. E. (1981). The measurement of experienced burnout. Journal of Organizational Behavior, 2(2), 99-113. https://doi.org/10.1002/job.4030020205

Moody, K., Kramer, D., Santizo, R. O., Magro, L., Wyshogrod, D., Ambrosio, J., Castillo, C., Lieberman, R., \& Stein, J. (2013). Helping the helpers: Mindfulness training for burnout in pediatric oncology - A pilot program. Journal of Pediatric Oncology Nursing, 30(5), 275-284. https://doi.org/10.1177/1043454213504497

Neff, K. D. (2003). Development and validation of a scale to measure self-compassion. Self and Identity, 2, 223-250. https://self-compassion.org/wpcontent/uploads/publications/empirical.article.pdf

Parker, P. A., \& Kulik, J. A. (1995). Burnout, self- and supervisor-rated job performance, and absenteeism among nurses. Journal of Behavioral Medicine, 18, 581-599. https://doi.org/10.1007/BF01857897

Saeidi R., Izanloo A., \& Izanlou S. (2020 A Study of the Relationship between Job Satisfaction and Burnout among Neonatal Intensive Care Unit Staff. Iranian Journal of Neonatology, 11(1), 67-70. doi: 10.22038/ijn.2019.39744.1634 
Sallon, S., Katz-Eisner, D., Yaffe, H., \& Bdolah-Abram, T. (2017) Caring for the caregivers: Results of an extended, five-component stress-reduction intervention for hospital staff. Behavioral Medicine, 43(1), 47-60. Doi: 10.1080/08964289.2015.1053426

Shrank, W. H., Patrick, A. R., \& Brookhart, M. A. (2011). Healthy user and related biases in observational studies of preventive interventions: A primer for physicians. Journal of General Internal Medicine, 26(5), 546-550. doi: 10.1007/s11606-010-1609-1

Slatyer, S., Craigie, M., Heritage, B., Davis, S., \& Rees, C. (2018). Evaluating the effectiveness of a brief mindful self-care and resiliency (MSCR) intervention for nurses: A controlled trial. Mindfulness, 9(2), 534-546. https://doi.org/10.1007/s12671-017-0795-x

Spence Laschinger, H. K., \& Leiter, M. P. (2006). The impact of nursing work environments on patient safety outcomes: The mediating role of burnout engagement. The Journal of Nursing Administration 36(5), 259-267. Retrieved from https://search.ebscohost.com/login.aspx?direct=true\&db=rzh\&AN=106317912\&authtype $=$ shib\&site $=$ ehost-live $\&$ scope $=$ site

Stamm, B.H. (2012). Professional Quality of Life: Compassion Satisfaction and Fatigue Version 5 (ProQOL). Retrieved from www.proqol.org

Thompson, C., Dalgleish, L., Bucknall, T., Estabrooks, C., Hutchinson, A. M., Fraser, K., ... Saunders, J. (2008). The effects of time pressure and experience on nurses' risk assessment decisions: A signal detection analysis. Nursing Research 57(5), 302-311. doi: 10.1097/01.NNR.0000313504.37970.f9

Vahey, D. C., Aiken, L. H., Sloane, D. M., Clarke, S. P., \& Vargas, D. (2004). Nurse burnout and patient satisfaction. Medical Care, 42(2), II57-II66. doi: 
10.1097/01.mlr.0000109126.50398.5a

van Mol, M. M. C., Kompanje, E. J. O., Benoit, D. D., Bakker, J., \& Nijkamp, M. D. (2015). The prevalence of compassion fatigue and burnout among healthcare professinoals in intensive care units: A systematic review. PLoS ONE, 10(8), e0136955. doi:10.1371/journal.pone.0136955

Wang, S., Wang, L., Shih, S., Chang, S., Fan, S., \& Hu, W. (2016). The effects of mindfulnessbased stress reduction on hospital nursing staff. Applied Nursing Research, 38(2017), 124-128. http://dx.doi.org/10.1016/j.apnr.2017.09.014 